\title{
ON THE ILIEFF-SENDOV CONJECTURE
}

\author{
JOHNNY E. BROWN
}

The well-known Ilieff-Sendov conjecture asserts that for any polynomial $p(z)=X T_{k=l}\left(z-z_{k}\right)$ with $\left|z_{k}\right|<1$, each of the disks. $\left|z-z_{k}\right| z$ $1(1 \leq k \leq n)$ must contain a critical point of $p$. This conjecture is proved for polynomials of arbitrary degree $n$ with at most four distinct zeros. This extends a result of Saff and Twomey.

1. Introduction. The Gauss-Lucas Theorem states that all the critical points of a polynomial $p(z)$ lie in the convex hull of its zeros. This is a result concerning the position of all the zeros of $p^{\prime}(z)$ relative to all the zeros of $p(z)$. Suppose we focus attention on any arbitrarily fixed zero of $p(z)$ and ask for the location of a zero of $p^{\prime}(z)$ relative to it. This leads to the well-known conjecture of Ilieff and Sendov [3; Problem 4.5] which asserts that if $p(z)$ has the form

$$
p(z)=f\left[\left(z_{k}-z_{k}\right), \quad \mid z_{k}+<l \quad(k<k<n)\right.
$$

then each of the disks $\backslash z-z_{k} \backslash<\backslash\{I$ shows that $k$, cpntains a zero of
$p^{\prime}(z)$. The polynomial $p(z)=z^{n}$. This conjecture is nearly a quarter of a century old and has been verified in some special cases, most notably if $p(z)$ has the form (1) and if

(A) $2 \leq n \leq 5[1,6,8]$,

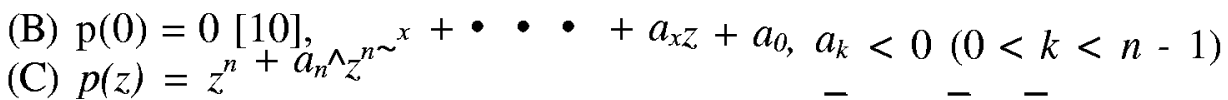
[11],

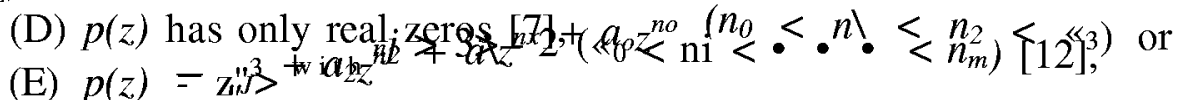
$P(z)=\mathrm{E}^{\wedge}=0 \mathrm{o}^{z j^{z}}$

(F) the vertices of the convex hull of the zeros of $p(z)$ all lie on $\langle z \backslash=1[10]$,

(G) the convex hull of the zeros of $p(z)$ is a triangular region [12],

(H) $p(z)=\left(\mathrm{z}-z_{x} r\left(z-z_{2} r\left(z-\mathrm{z}_{3}\right)^{13} \quad[9]\right.\right.$.

The last case $(\mathrm{H})$, due to Saff and Twomey, states that the conjecture is true for any polynomial of the form (1) with at most three distinct 
zeros. The purpose of this paper is to establish the conjecture for any polynomial with at most four distinct zeros.

Observe that $(\mathrm{H})$ follows immediately from $(\mathrm{G})$. A problem posed by Schmeisser [12] related to $(G)$ is to determine whether the conjecture is true if the convex hull of the zeros of $p(z)$ is a quadrangular region. Our result verifies a special case of his problem.

2. Main results. We can now state our main result.

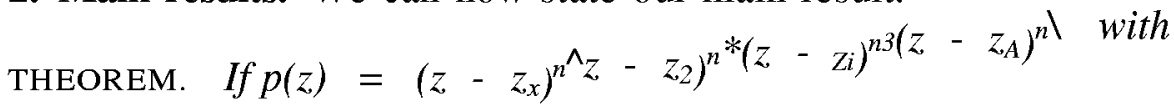
$\mid z k \backslash \leq 1 \quad(1 \leq £ \leq 4)$, then each of the disks $\mid z-z_{k} \backslash<1 \quad(1 \leq k \leq 4)$ contains a zero ofp' $(z)$.

Before embarking on the proof we briefly illustrate the idea by giving a simple proof of the Saff-Twomey result. Suppose $p(z)=$
$(z-\mathrm{Zi})^{\prime \prime \prime}\left(\mathrm{z}-z_{2}\right)^{\text {ni }}\left(z-z^{-} \wedge^{d}\right.$

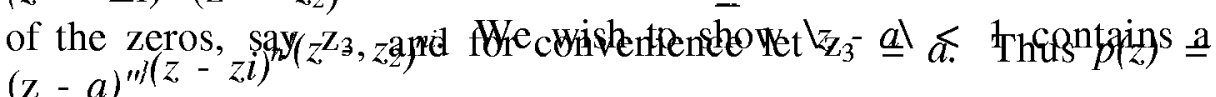
zero of $p^{\prime}(z)$. If $\ll_{3}>1$ we are done, so suppose $\ll_{3}=1$. It is then clear that $p^{\prime}(z)=n\left(z-z_{1}\right)^{n_{1}-1}\left(z-z_{2}\right)^{n_{2}-1}\left(z-\zeta_{1}\right)\left(z-\zeta_{2}\right)\left(n_{1}+n_{2}+1=n\right)$ and if we let $q(z)=p(z) /(z-a)$ then also $p^{\prime}(z)=(z-a) g^{\prime}(z)+q(z)$. Hence we see that $p^{\prime}(a)=g(a)$ and so

$$
n\left(a-z_{x} r^{x}\left\{a-z_{2}\right)^{n} \backslash a-\mathrm{C},\left(\mathrm{a}-\mathrm{C}_{2}\right)=(a-\mathrm{z},)^{\prime \prime}\left(\mathrm{a}-z_{2}\right)^{n} \backslash\right.
$$

We may suppose that $\mathrm{V} a-\mathrm{Ci} \mid \leq \mathrm{b}-\mathrm{C}{ }^{\text {and }}{ }^{\text {get }}$

$$
n|a-\mathrm{C}|^{2} \leq|a-z \backslash \backslash| a-z_{2} \mid \leq 4 .
$$

Hence $\backslash a-\mathrm{Ci} \mid \leq 2 / \wedge / \bar{n}$. If $n \geq 4$, we are done; while if $n=2$ or 3 we already know the conjecture is true. The proof of the theorem is based on this simple idea, however we need some preliminary results first.

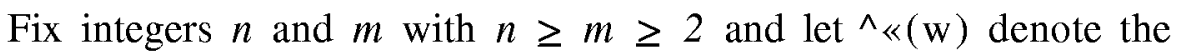
class of all monic polynomials of degree $n$ with at most $m$ distinct zeros in $|z| \leq 1$ :

$$
p(z){ }_{k=l}^{m} H\left(z-z_{k} r, \quad \mid z_{k} \backslash<\backslash \quad(l \leq k \leq m),\right.
$$

where $n_{k}>1$ and $\mathrm{XT}=\mathrm{i}^{n k}={ }^{n-}$ From (2) we get

$$
\left.p^{\prime}(z)=n \sum_{. k=1}^{m}\left(z-z_{k}\right)^{n_{k}-1}\right]\left[\prod_{j=1}^{1}\left(z-\zeta_{j}\right)\right] \text {. }
$$


Let

$$
\begin{gathered}
I\left(z_{k}\right)= \begin{cases}\min _{1 \leq j \leq m-1}\left|z_{k}-\zeta_{j}\right|, & \text { if } n_{k}=1, \\
0, & \text { if } n_{k}>1,\end{cases} \\
I(p)=\max _{l<k<m} I\left(z_{k}\right) \text { and } I\left(\&>_{n}(m)\right)=\sup _{p \in \Theta_{n}(m)} I(p) .
\end{gathered}
$$

The IliefF-Sendov conjecture says that $I\left(\&>_{n}(m)\right)<1$.

LEMMA 1. (i) There exists an extremalpolynomial $p^{*} \mathrm{e}^{\wedge} \ll(\mathrm{w})$ such that $I\left(p^{*}\right)=I\left(\mathscr{P}_{n}(m)\right)$.

(ii) / ?* has a zero on each subarc of $k 1=1$ oflength $n$.

This lemma is essentially proved in [5, 7] but for completeness sake and a slightly easier proof we present it here.

Proof. Since $\nmid p(z) \leq(1+r)^{n}$, where $\backslash \backslash \backslash \leq r$, for any $p$ e $\mathscr{P}_{n}(m)$, it is clear that $3 P_{n}(m)$ is a normal family in C. Each polynomial in $3^{\circ}{ }_{n}(t n)$ is monic and has at most $\mathrm{m}$ distinct zeros and hence ${ }^{\wedge}(\mathrm{m})$ is compact. By definition there exists a sequence $\left\{p^{\wedge}\right\}<Z 3 P_{n}($ rri $)$ such that $I($ pic $) \longrightarrow l\left(S^{\wedge}{ }_{n}(m)\right)$ as $k-$ oo. Choose a convergent subsequence (call it $\{P k\}$ again) so that $P k-* P^{*}$ uniformly on compact subsets of C. Let

$$
p^{*}(z)=\prod_{k=1}^{m}\left(z-z_{k}^{*}\right)^{n_{k}^{*},} \quad\left|z_{k}^{*}\right| \leq 1, \quad \sum_{k=1}^{m} n_{k}^{*}=n
$$

and so

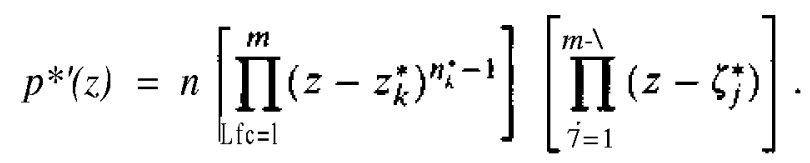

Assume $/\left(\mathrm{p}^{*}\right)<I\left(\mathscr{P}_{n}(m)\right)$. Then $I\left(p^{*}\right)=I\left(3^{\circ}{ }_{n}(m)\right)-3 \mathrm{e}$, for some

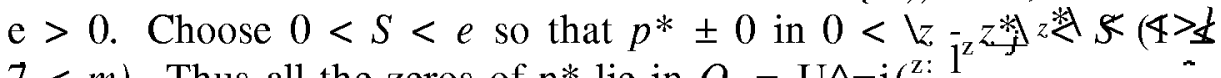
$7 \leq m)$. Thus all the zeros of $p^{*}$ lie in $Q,=\mathcal{U}^{\wedge}=\mathrm{i}\left({ }^{z}\right.$ : and (by definition of $/\left(/ ?^{*}\right)$ ) at least one zero of $p^{* \prime}$ lies in each of the disks $\mid z-z_{j} \backslash \leq I\left(p^{*}\right)(I \leq j \leq m)$. By Hurwitz' Theorem, for all $k \geq K Q$ sufficiently large, all the zeros of $p_{k}$ lie in $\mathrm{Q}$ and each disk $\checkmark z-z^{*} \backslash \leq I\left(p^{*}\right)+e$ will contain a zero of $p_{k}^{\prime}$. Hence we see that $\left.I(P k) \wedge H P^{*}\right)+e+S<I\left(p^{*}\right)+2 e=I\left\{3^{\circ}{ }_{n}(m)\right)-e$. Letting $k-$ oo we get $I\left(3^{\circ}{ }_{n}(m)\right)<I\left({ }_{n}(m)\right)-e$ and a contradiction is reached. This proves (i). 
To prove (ii) we first assert that the extremal polynomial $p^{*}$ of the form (4) must have at least one zero on $|z|=1$. Suppose not. Then $r=\max \underline{j}_{-}^{\wedge}\left|z_{k}\right|<1$. Define $\hat{p}$ by

$$
\left.\tilde{p}(z)=\frac{1}{r^{n}} p^{*}\right) r z=\prod_{k=1}^{m}\left(z-\frac{z_{k}^{*}}{r}\right)^{n_{k}^{*}} .
$$

Clearly $\tilde{p}$ e $t P r S j r r i)$ and since $\tilde{p}^{\prime}(z)=-p h r P^{* \prime}(r z)$, we see that

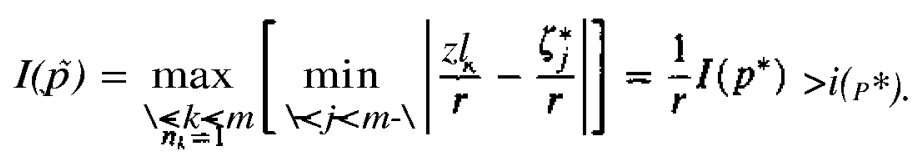

Contradiction.

Next, we assert that $p^{*}$ must have at least two distinct roots on $|z|=1$. Suppose not. Then $p^{*}$ has the form (4), with say $\left|z^{\wedge}\right|=1$ and $\left\langle z^{*} \backslash<1(1 \leq k \leq m-1)\right.$. By a rotation, we can assume $z^{*}{ }_{m}=1$. Let $5=(1-\mathrm{r}) / 2$, where $r=\operatorname{maxj} \wedge \wedge, \wedge ! \mid z^{*} k$. If $p(z)=p^{*}(z+s)$, then again $\tilde{p} € \wedge \ll(\mathrm{m})$ and $I\left(p^{*}\right)=I\left(p^{*}\right)$. Hence $p$ is also extremal. However, it is easy to see that its zeros $z_{k}^{\tilde{k}}=z^{*}{ }_{k}-s$ satisfy $\left|\mathrm{z}^{\wedge}.\right|<1$ $\wedge \leq k \leq m)$. Contradiction.

We have shown that the extremal polynomial $p^{*}$ has the form (4) with $\left|z^{\wedge} \backslash-\right| z^{*}{ }_{m-x} \backslash=1$ and $z^{*}{ }_{m} \wedge z^{*}$

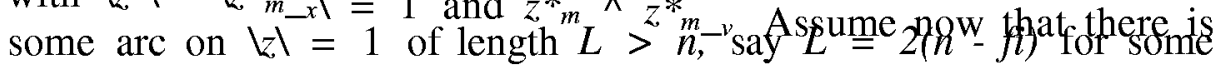
$0</ ?<7 \mathrm{r} / 2$, on which $p^{* \wedge} 0$. By a rotation, we may suppose that $z^{*}{ }_{m}=e^{i a}$ and $z^{*}{ }^{*} x=e^{i a}$ for some $0<a<7 ?<n / 2$. (Thus $p^{*}\left(e^{e}\right)^{m} \overline{\overline{0}}$ for $\mathrm{a}<\mid \Gamma^{-x}<\wedge$. $\Gamma^{\sim}$ By relabeling the zeros suppose that $\left|z\left\|\leq|z \| \backslash \leq \cdots \leq| z^{*} J<\overline{1}\right.\right.$ and $|z ;+1|=\cdot \cdot \cdot=\left|z^{*} m_{-2}\right|=1$ (put $n_{Q}-0$ if all the zeros of $p^{*}$ lie on $\langle\bar{z}=1$ ). Define $r$ and 5 as follows:

$$
r \sim \mathbf{j} \underset{\max }{\mathbf{J}}|z \mathbf{~ 0 f}|, \quad \text { if } \ll_{0}=\mathbf{0},
$$

and

$$
s=\min \left\{\cos a, \frac{1-\mathrm{r}}{2}\right\}
$$

Again consider $\tilde{p}(z)=p^{*}(z+s)$ and note that $\tilde{p} €^{\wedge}(\mathrm{m})$ with $\left./(/)\right)=$ $I\left(p^{*}\right)$. An easy check shows that the zeros $z^{\wedge}=z_{k}^{*}-s$ of $\tilde{p}$ all lie in $|z|<1$. Contradiction. This completes the proof of the lemma.

LEMMA 2. The Ilieff-Senglovahgniectune isf true if

(i) $p(z)=$ "Uk=i(

(ii) $\left.p(z)=\left(\mathrm{z}^{-}-\mathrm{e}^{\prime} 0^{\circ}\right)^{2} U l l^{\wedge} \bar{z}-z_{k}\right)$ and $2 \leq n<L_{-}\left(d_{0} € \mathrm{R}\right)$. 
The first part of this lemma is known and there are various proofs, but the proof of (ii) gives (i) along the way so we include it here. This now makes the paper completely self-contained.

Proof. Let $>(\mathrm{z})=\mathrm{n}^{*}=\mathrm{i}\left(*{ }^{z} k\right)$ with $\left|z_{k}\right\rangle \leq 1$. Then $p^{\prime}(z)=$ $n$ YYJZi $\left(^{z} \sim C j\right)$ - Distinguish one of the zeros, say $z_{n}$ (call it $a$ ) and by a rotation let

$$
\begin{aligned}
& \mathrm{n}-\mathrm{i} \\
& p(z)=\{z-a) \vee\left(z-z_{k}\right), \quad 0 \leq a<l,-\quad|z+k|<1
\end{aligned}
$$

We may also suppose that $0<a<1$. If a $=0$, the conjecture is trivially true for this zero. If $a=1$, we are also done. Indeed, if we let $q(z)=Y l_{-}^{n} Z \backslash\left(z-z_{k}\right)$. Then we see that $p^{\prime \prime}(\Lambda) / p^{\prime}(\Lambda)=2 q^{\prime}(l) / q(l)$ and so

$$
\sum_{j=1}^{n-1} \frac{i}{1-\zeta_{j}}=\sum_{k=1}^{n-1} \frac{\hat{L}}{1-z_{k}}
$$

Supposing $\operatorname{Re}\{1 /(1-\mathrm{Ci})\}_{-}>\operatorname{Re}\{1 /(1-\mathrm{Cy})\}_{-}(l \leq j<n-l) a \&$ we may, then from (6) we get

$$
(n-1) \operatorname{Re}\left\{\frac{1}{1-\zeta_{1}}\right\} \geq 2 \sum_{k=1}^{n-1} \operatorname{Re}\left\{\frac{1}{1-z_{k}}\right\} \geq(n-1) .
$$

Hence $\operatorname{Re}\{1 /(1-\mathrm{Ci})\} \geq 1$ or $\mid \mathrm{d}-\lambda<\leq$ so certainly $|\mathrm{Ci}-1|<1$ (cf. [2]). Hence suppose $0<a<1$.

If we put $z=T(w)=(w-a) l(a w-1)$, we have

$$
p(T(w))=\tilde{p}(w)(a w-1)^{-n},
$$

where

$$
{ }^{*} p(w)=A w\left[w^{n} \sim^{l+b n-i i v " \nu^{2}+\cdots+* !}\right] .
$$

From (7), the zeros of $\tilde{p}(w)$ are $0, W, W 2, \ldots, w_{n-\backslash}$ where $w_{k}=$ $T \sim^{l\left(z_{k}\right)}(1<k<n-1)$. Thus we get

$$
\left|b_{1}\right|=\prod_{k=1}^{n-1}\left|w_{k}\right| \leq 1
$$

and

$$
\mathbf{I V i l}=\left|\sum_{k=1}^{\mathrm{n}-1} w_{k}\right|-<n-\mathrm{l}
$$


Differentiating (7) gives

$$
\frac{d p\{T(w))}{d w}=\frac{d p\{T(w))}{d z} \frac{d z}{d w}=-a(a w-l) \sim^{n}-{ }_{l} D_{l / a p}(w)
$$

where

$$
D_{1 / a} \tilde{p}(w)=n p(w)+(\mathrm{I} / \mathrm{a}-w) p^{\prime}(w)
$$

is the polar derivative of $p$ with respect to $1 / a$ (see Marden [4]). Hence we arrive at

$$
p^{\prime}(T(w))=-a(a w-l)-\gg-1 \frac{d w}{d z} D_{1 / a} \hat{p}(w)
$$

(where' denotes differentiation with respect to $\mathrm{z}$ ). A brief calculation using (8) and (11) gives

$$
D_{l / a} \tilde{p}(w)=B\left[w^{\mathrm{n}-1}+\cdots+\left(\frac{b_{1}}{n+a b_{n-1}}\right)\right]=B \prod_{k=1}^{n-1}\left(w-\gamma_{k}\right),
$$

where $\left.\left|y_{x}\right| \leq\left|y_{2} \backslash \prec \cdots \prec\right| y_{n-i}\right\rangle$ - It follows from (9), (10) and (13) that

$$
\prod_{k=1}^{n-1}\left|\gamma_{k}\right|=\left|\frac{b_{i}}{n+a b_{n-1}}\right| \leq \frac{1}{n-a\{n-1)^{\prime}}
$$

Let us now suppose that $y y \backslash \leq f i$. Then from (12) we have

$$
D l / a P(V l)=\underset{a}{\wedge}\left(a \gamma_{1}-1\right)^{n+1}>\wedge P>\left(T_{(n)}\right)=\boldsymbol{o} .
$$

Hence $/ /(\mathrm{Co})=0$, where $\mathrm{Co}=T\left(y_{x}\right)$ and so $p^{\prime}(z)$ has a zero $\mathrm{Co}{ }^{\text {such }}$ that

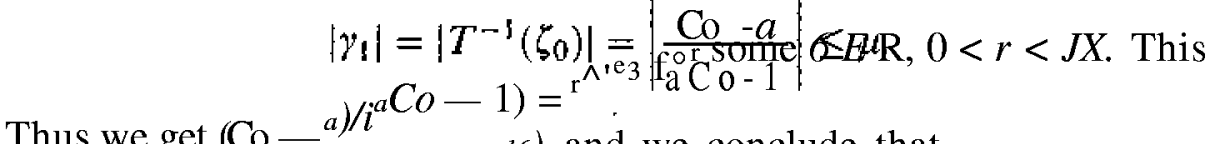

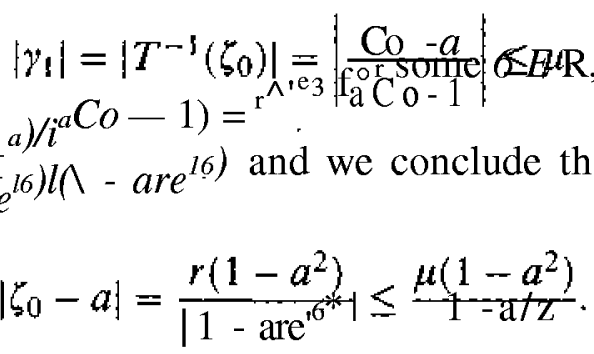

Hence if $\mu\left(1-a^{2}\right) /(1-a \mu) \leq 1$, or equivalently,

$$
\mu<\frac{1}{1+a-a^{2}}
$$

then $\mathrm{Co}-o \backslash \_<1$. It remains to show that (15) holds for the cases stated in the lemma. 
Suppose first that $2 \leq n \leq 4$. Then from (14) we have

$$
\left|\gamma_{1}\right| \leq\left[\frac{1}{n-a(n-)_{m}}\right]^{1 /(n-1)} \equiv \mu
$$

It is easy to check that (15) holds for this $f i$ and $2 \leq n \leq 4$ (and any $0<a<1$ ). This proves (i).

Suppose next that $p(z)$ has the form stated in $\left.{ }_{l}(\dot{\phi} \dot{\mathrm{i}})\right)=\mathrm{T}_{\mathrm{e}}$ ) we conclude that $p(w)$ has a double root at $T \sim^{l}$

$U Q$ e R). From (11) we see that $y_{n-} \backslash=e^{m}$ is a zero of $D \searrow_{a p}(w)$. Using (14) we have the following

$$
\prod_{k=1}^{n-l} \mathbf{n i t i}-\operatorname{nitt}_{k=1}^{n-1} K \frac{1}{\left.n-a_{\{}^{\{} n-1\right)}
$$

and so

$$
\left|\gamma_{1}\right| \leq\left[\frac{1}{n-a(n-\mathrm{I})}\right]^{1 /(n-2)} \equiv \mu
$$

For this $n$ and $2 \leq n \leq 7$, it is simple to check that (15) holds for any $0<a<1$.

We should point out that this proof can be slightly modified to give a proof of Laguerre's Theorem (see [4] for another proof).

Proof of theorem. For fixed $n \geq m \geq 3$, let $p(z)$ be of the form (2). From Lemma 1, an extremal polynomial for $3^{\circ}(m)$ exists and without loss of generality we may assume $p$ is extremal i.e., $I(p)=I^{\wedge}$ nim)). Distinguish one of the zeros, say $z_{m}$ and let $z_{m}=a$. We may suppose too that $0 \leq a \leq 1$. We want to show $|z-a\rangle \leq 1$ contains a critical point of $p$. If $n_{m}>1$, we are done so suppose $n_{m}=1$. Thus we have

$$
p(z)=(z-a) \prod_{k=1}^{m-1}\left(z-z_{k}\right)^{n_{k}}, \quad \backslash z_{k}<\backslash, \quad \sum_{A:=1}^{m-1} n_{k}=n-1
$$

and

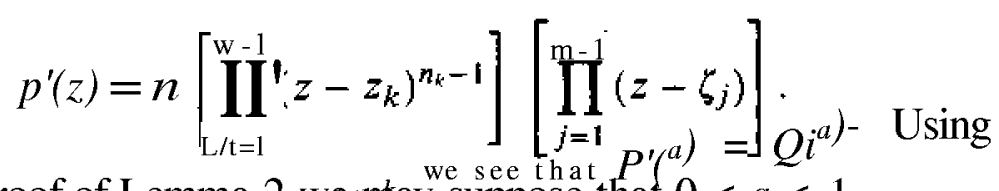

As in the proof of Lemma 2 wemkay suppose that $0<a<1$.

If we let $q(z)=Y l^{\wedge} l i^{2}$

(16) and (17), we obtain after cancellations

$$
n \prod_{j=1}^{m-1}\left(a-\zeta_{j}\right)=\prod_{\mathrm{A}:=1}^{m-1}\left(a-z_{k}\right)
$$


Assuming that $\backslash a-f \mathrm{i}|\leq \backslash a-C\rangle(1 \leq j \leq m-1)$, as we may, we get

$$
n \backslash a \quad \mathrm{CiT}_{k=i}^{\prime 1} \quad\left\langle Y \backslash \backslash a-z_{k}\right|<\backslash a-z_{x} \| l a-\quad z_{2} \backslash 2^{m \sim}
$$

Hence we have the estimate

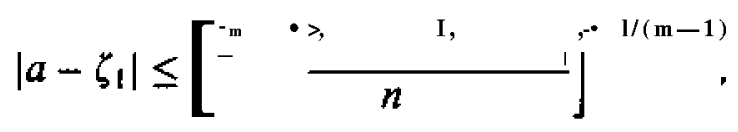

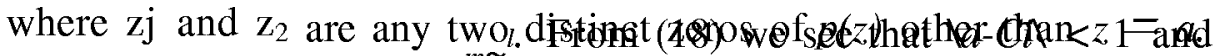

Suppose first that $n \geq 2^{m \sim}$

we are done. Hence suppose $2<n<2^{m \sim}$

Now we restrict ourselves to $m=4$. Thus we need only consider polynomials of the form (16) of degree $2 \leq n<8$. By Lemma 2(i) we need only consider $n=5,6$ and 7 . Thus, our polynomial has the form

$$
p(z)=(z-a)\{z-z,)^{\prime \prime}\left(\left(^{*}-z i Y^{\wedge} z-z i\right)^{n} \backslash\right.
$$

where $0 \leq n \backslash \leq n_{2} \leq n^{\wedge}$ and $r i+n_{2}+n^{\wedge}=n-1$. Since $«=5,6$ or 7 , we must have $n^{\wedge} \geq 2$ in each case. Recall that $p(z)$ is an extremal polynomial for $3^{\circ}(m)$ and hence by Lemma 1 it will have a zero on each subare of $\langle z\rangle=1$ of length $n$. We are thus led to two cases:

Case 1. $\mathrm{p}(\mathrm{z})$ has exactly two distinct zeros on $\langle z\rangle=1$.

Care 2. $\mathrm{p}(\mathrm{z})$ has more than two distinct zeros on $\langle z\rangle=1$.

Case 2 is easily disposed of as follows. In this case (recall $0<a<1$ ),

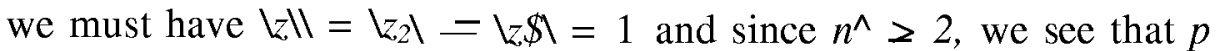
has a zero of order two on $\langle z \backslash=1$. Since $n \leq 7$, we may apply Lemma 2(ii) and we are done.

In order for Case 1 to hold, the two zeros must be negatives of each other, say $Z Q$ and $-Z Q$. Making use of the estimate (18) we find that

$$
\left|a-\zeta_{1}\right| \leq\left[\frac{2\left|a-z_{0}\right|\left|a+z_{0}\right|}{n}\right]^{1 / 3} \leq\left[\frac{4}{n}\right]^{1 / 3}
$$

and so $\backslash a-\mathrm{Cil} \leq 1$ for $n \geq 4$. By Lemma 2(i), the conjecture is true for $n=2$ and 3 . The proof of the theorem is complete.

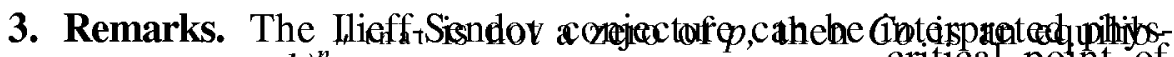
ically in terms of $k$ f $^{n}$ rce fields. Indeed, if $\mathrm{Co}$ is a critical point of $P\left({ }^{z}\right)=\operatorname{Iir}=\mathrm{i}($

rium point in the plane field in which the force exerted on a particle by a point charge at $z_{k}$ (with charge $n^{\wedge}$ ) is inversely proportional to its distance from $z_{k}$. The Ilieff-Sendov conjecture asserts that either the 
disk of radius one about each zero $z_{k}$ contains at least one of these equilibrium points or else $n_{k}>1$. In general these cases are mytually exclusive. For example if $p(z)=(z-1)^{3(z-/)}$, then the disk $|z-1| \leq 1$ contains no such equilibrium point. Since the conjecture is trivially true for the zero $z_{k}$ if $n_{k}>1$, the conjecture is only interesting if $z_{k}$ is a simple zero. In this case we are led to the problem of determining just how close a critical point can then be to a simple zero. We thus pose the following problem:

Let $Q_{n}$ denote the set of all polynomials for the form $P(z)=z n^{n_{k} Z} \backslash\left(z-z_{k}\right)$, with $\left.z_{k}\right\rangle>1(1 \leq k \leq n-1)$.

Determine the largest constant $c_{n}>0$ such that $p^{\prime}(z)^{\wedge}$ 0 in $\backslash z \backslash<c_{n}$ for all $p E Q_{n}$.

Clearly $c_{n}<1$ and we expect that the critical point nearest the zero $z=0$ is an equilibrium point in the force field as described above. By concentrating all the charge at one point, together with a single charge at $z=0$, it is $\mathrm{s}^{\mathrm{s}}$ keasonable to conjecture that $c_{n}=$ Jin (consider $p(z)=z \cdot\left(z-e^{i e}\right)$

Finally, we must point out that recently a proof of the Ilieff-Sendov conjecture was announced (by title) in the Abstracts of the American Mathematical Society (June 1986) by V. I. Istrătescu. He claims his method uses a notion called "bare points" and "a Krein-Milman theorem". The proof of our main result is completely self-contained, relies on classical methods and indicates that a proof of the full conjecture must be delicate in nature.

\section{REFERENCES}

[1] D. A. Brannan, On a conjecture of I lieff, Proc. Cambridge Philos. Soc, 64 (1968), 83-85.

[2] A. W. Goodman, Q. Rahman and J. Ratti, On the zeros of a polynomial and its derivative, Proc. Amer. Math. Soc, 21 (1969), 273-274.

[3] W. K. Hayman, Research Problems in Function Theory, London (1967).

[4] M. Marden, Geometry of Polynomials, Amer. Math. Soc, (1966).

[5]_ Conjectures on the critical points of a polynomial, Amer. Math. Monthly, 90(1980), 267-276.

[6] A. Meir and A. Sharma, On Ilyejf's conjecture, Pacific J. Math., 31 (1969), 459-467.

[7] D. Phelps and R. Rodriguez, Some properties of extremal polynomials for the Ileiff Conjecture, Kodai Math. Sem. Report, 24 (1972), 172-175.

[8] Z. Rubinstein, On a problem ofllyeff, Pacific J. Math., 26 (1968), 159-161.

[9] E. B. Saff and J. Twomey, A note on the location of critical points of polynomials, Proc. Amer. Math. Soc, 27 (1971), 303-308. 
[10] G. Schmeisser, Bermerkungen zueiner Vermutung von Ilieff, Math. Z., I11 (1969), 121-125.

[11]_, Zur Lage der kritischen Punkte eines Polynoms, Rend. Sem. Mat. Univ. Padova.

[12]__ On Ilieff's conjecture, Math. Z., 156 (1977), 165-173.

Received June 9, 1987. Some of this work was done while the author was on sabbatical leave at the University of Delaware.

PURDUE UNIVERSITY

WEST LAFAYETTE, IN 47907 